\title{
The conjunction fallacy and the many meanings of and
}

\author{
Ralph Hertwig ${ }^{\mathrm{a}, *}$, Björn Benz ${ }^{\mathrm{b}}$, Stefan Krauss ${ }^{\mathrm{c}}$ \\ ${ }^{a}$ Department of Psychology, University of Basel, Missionsstrasse 60/62, 4055 Basel, Switzerland \\ ${ }^{\mathrm{b}}$ University of Lüneburg, Germany \\ ${ }^{\mathrm{c}}$ University of Kassel, Germany
}

\section{A R T I C L E I N F O}

\section{Article history:}

Received 25 September 2007

Revised 9 April 2008

Accepted 29 June 2008

\section{Keywords:}

Conjunction fallacy

Pragmatic and semantic inferences

Rationality

\begin{abstract}
A B S T R A C T
According to the conjunction rule, the probability of A and B cannot exceed the probability of either single event. This rule reads and in terms of the logical operator $\wedge$, interpreting $A$ and $B$ as an intersection of two events. As linguists have long argued, in natural language "and" can convey a wide range of relationships between conjuncts such as temporal order ("I went to the store and bought some whisky"), causal relationships ("Smile and the world smiles with you"), and can indicate a collection of sets rather than their intersection (as in "He invited friends and colleagues to the party"). When "and" is used in word problems researching the conjunction fallacy, the conjunction rule, which assumes the logical operator $\wedge$, therefore cannot be mechanically invoked as a norm. Across several studies, we used different methods of probing people's understanding of and-conjunctions, and found evidence that many of those respondents who violated the conjunction rule in their probability or frequency judgments inferred a meaning of and that differs from the logical operator $\wedge$. We argue that these findings have implications for whether judgments involving ambiguous and-conjunctions that violate the conjunction rule should be considered manifestations of fallacious reasoning or of reasonable pragmatic and semantic inferences.
\end{abstract}

(c) 2008 Elsevier B.V. All rights reserved.
... it is a mistake to analyze natural language words like and as being identical to entities of the man-made logical terminology which so clearly derives from natural language (rather than the other way around) and so clearly has needs and purposes distinct from those of natural language (Sweetser, 1990, p. 92).

One of the most frequent words in the English language is and. In Leech, Rayson, and Wilson's (2001) analysis of word frequencies in a 100 million word collection of samples of written and spoken language (the British National Corpus) the unremarkable three-letter word "and" is the third most frequent word. Its commonness and plainness, however, do not mean that the most general connective in the English language lacks the ability to convey a wide range of relationships between the states of affairs described by the conjuncts. In fact, one reason for linguists'

\footnotetext{
* Corresponding author. Tel.: +4161267 0611; fax: +41612670441. E-mail address: ralph.hertwig@unibas.ch (R. Hertwig).
}

enduring fascination with and is that among all coordinating conjunctions (e.g., or, but, nor) it has the least semantic and syntactic limits, the least specific meaning, and the highest context dependency (Lang, 1991).

Take, for instance, the use of and in (1):

(1) Her husband is in hospital and she is seeing other men.

As Kitis (2000) pointed out, the and in (1) does much more than conjoin the two clauses. Rather, it functions as an "emotional device" that communicates the speaker's emotional attitude, surprise, or even outrage.

The notion of and's context dependency stands in stark contrast with the highly constrained meaning of the logical and operator $(\wedge)$. The logical $\wedge$ represents the Boolean intersection of two sets. For illustration, if set $A$ contains all even numbers (multiples of 2 ) in the universe and set $B$ contains all multiples of 3 , then the intersection of the two sets - all elements in set $A \wedge B$ - will be multiples of 
six. Compare this logical meaning to the use of and in (2) and (3):

(2) We have invited friends and colleagues to the party.

(3) John and Mary lifted the piano.

What (2) and (3) have in common is that and sets the conjuncts "friends" and "colleagues," and "John" and "Mary" side-by-side as coequal pieces of a sum of entities. Thus, it is, for instance, the union or addition of friends and colleagues who are invited to the party and not the intersection of both sets of people (colleagues who are also friends or vice versa); in logic this union would be represented by the or (" $\vee$ ") operator. As the linguist Hurford (1987) pointed out, "the commonest indicators of addition" across languages such as Arabic (wa), English (and), French (et) and German (und), "are words which also happen to indicate the logical conjunction of propositions" (p. 226). Moreover, several linguists have proposed distinguishing two ands in English (Partee \& Rooth, 1983; Keenan \& Faltz, 1985). Keenan and Faltz, for instance, proposed a distinction between the "lower order and we have been using which forms intersections and a higher order one which (roughly) forms sets" (p. 270).

If there are indeed these two versions of and in natural language, people's reading of a sentence will depend on which and they assume. Moreover, depending on which and they assume, people may arrive at nearly opposite understandings of a sentence, depending on whether they interpret the connective as referring to an intersection, a union of sets, or, as we discuss in the second part of the paper, to a causal relationship. We aim to show that many of those respondents who violate the conjunction rule infer a meaning of and that is different from the logical operator $\wedge$. As a consequence, what appear to be manifestations of fallacious reasoning can, alternatively, be understood as reasonable inferences about the wide range of relationships between the conjuncts that the natural language and can convey. In addition to the ambiguity of the and connective, we also address the potential ambiguity caused by a ranking response mode. We begin by reviewing the conjunction fallacy, a prominent deviation between people's probabilistic reasoning and a law from probability theory.

\section{The and in research on the Linda task: Logical operator or natural language conjuction?}

In their seminal article on the conjunction fallacy, Tversky and Kahneman (1983) distinguished between two experimental paradigms: the $M \rightarrow A$ and the $A \rightarrow B$ paradigms. The word problem that became the pivotal implementation of the $M \rightarrow A$ paradigm is the Linda task. Here, respondents read a brief personality sketch of a person, Linda. The sketch described her as an educated, outspoken, social activist. Based on this information, respondents were asked to rank eight statements about Linda according to their probability. Among them were "Linda is a bank teller" and "Linda is a bank teller and is active in the feminist movement". This task exemplifies the structure of the $M \rightarrow A$ paradigm, which consists of a causal model $M$ (Linda's persona), an event $B$ (bank teller) that is unrepresentative of the $M$, and an event $A$ (active feminist) that is highly representative of $M$. Most respondents (85\%) in Tversky and Kahneman's original study ranked $B$ \& $A$ as more probable than $B$, violating the conjunction rule. This rule states that the mathematical probability of a conjoint event cannot exceed the probability of any of its constituent events, that is, $p(\mathrm{~A} \wedge \mathrm{B}) \leqslant p(\mathrm{~A}), p(\mathrm{~B})$.

Tversky and Kahneman (1983) argued that participants based their judgments on the representativeness heuristic: Linda was judged as more likely to be a bank teller and active in the feminist movement rather than a bank teller, because she resembled a prototypical feminist bank teller more than she resembled a prototypical bank teller. This thesis and the interpretation of people's responses as a fallacy in probabilistic reasoning have sparked considerable controversy among psychologists and beyond (e.g., Adler, 1984, 1991; Dulany \& Hilton, 1991; Gigerenzer, 1991, 1996; Hintikka, 2004; Levi, 2004; Moldoveanu \& Langer, 2002; Politzer \& Noveck, 1991).

A number of researchers, starting with Tversky and Kahneman (1983), found that violations of the conjunction rule were markedly reduced (Fiedler, 1988) and sometimes even disappeared (Hertwig \& Gigerenzer, 1999) when information and questions were presented in the form of frequencies. For example, Hertwig and Gigerenzer gave participants a task describing 200 women who fitted the description of Linda. Participants were asked questions such as "How many of the 200 women are bank tellers and are active in the feminist movement?" No participant violated the conjunction rule (Study 3 in Hertwig and Gigerenzer). They argued that in problems such as the Linda paradigm, semantic inferences about the meaning of the polysemous term "probability" contribute to this finding. Specifically, most people appear to infer nonmathematical meanings of probability (such as "possibility" or "conceivability"; Fig. 1a in Hertwig and Gigerenzer), whereas the terms frequency and how many narrow down the possible spectrum of meanings to those that follow mathematical probability (Fig. 2 in Hertwig and Gigerenzer).

To demonstrate that the conjunction fallacy can nevertheless even occur in frequency representations, Kahneman and Tversky (1996) presented three groups of participants with the following frequency representation of the Linda problem:

Linda is in her early thirties. She is single, outspoken, and very bright. As a student she majored in philosophy and was deeply concerned with issues of discrimination and social justice.

Suppose there are 1000 women who fit this description. How many of them are
(a) High school teachers? [Groups 1, 2, 3]
(b) Bank tellers? [Groups 1, 2]
(c) Bank tellers and active feminists? [Groups 1, 3]

Group 1 estimated the number of women who were bank tellers, bank tellers and active feminists, or high school teachers. This group allowed for a within-subjects test of violations of the conjunction rule. Group 2 estimated the number of women who were either bank tellers or high 
school teachers, and Group 3 estimated the number of women who were either bank tellers and active feminists or high school teachers. The comparison of Groups 2 and 3 allowed for a between-subjects test. The majority of participants in Group 1 (64\%) gave judgments consistent with the conjunction rule. In contrast, the median estimate for bank tellers and active feminists (in Group 3) was larger than the median estimate for bank tellers (in Group 2) - a violation of the conjunction rule in a frequency formulation (henceforth we refer to this finding in a between-subjects design as a conjunction effect, as did Mellers, Hertwig, \& Kahneman, 2001).

Why did it occur? Did it occur because people relied on the representativeness heuristic as Kahneman and Tversky (1996) argued or, as Hertwig (in Mellers et al., 2001) argued, because the and-conjunction in this frequency formulation is ambiguous and can be read to mean (4) or (5) ${ }^{1}$ :

(4) How many of the 1000 women are both bank tellers and active feminists?

(5) How many of the 1000 women are bank tellers or active feminists?

If people understood "bank tellers and active feminists" to refer to the intersection (as in 4), subjects' estimates in Group 3 would violate the conjunction rule. However, if people understood "bank tellers and active feminists" to refer to the collection of both conjuncts (as in 5), sets, subjects' estimates in Group 3 would not represent an instance of the conjunction fallacy.

Mellers et al. (2001) conducted several tests of this explanation, using the strategy of studying less ambiguous conjunctive phrases such as feminist bank tellers, avoiding the connective altogether. It seems fair to conclude that the evidence Mellers et al. obtained was mixed: specifically, as long as filler items were included (e.g., high school teachers), conjunction effects persisted even with less ambiguous conjunction phrases (Experiments 1 and 3). However, conjunction effects disappeared when filler items were removed (see Experiment 2; for possible explanations see Hertwig and Kahneman's discussions in Mellers et al.). In his discussion of the mixed results, Kahneman suggested that the "initial issue on which we disagreed - whether respondents interpret 'and' as a union operator - can be tested by directly asking them to indicate their chosen interpretation on a Venn diagram" (Mellers et al., p. 275). Our first experiment in a series of four studies was designed to this end. Specifically, we probe people's understanding of the and connective and investigate whether those people, should there be any, who report a union interpretation arrive at systematically higher estimates than those who report an intersection interpretation.

Before we turn to this experiment, however, let us briefly review Bonini, Tentori, and Osherson's (2004) Experiment 2 that also pertains to the ambiguity of the and connective. Participants were asked to bet on one of

\footnotetext{
1 Note that this ambiguity did not occur in the original single-event probability formulation of the Linda task (Tversky \& Kahneman, 1983).
}

three statements of the form $p, p$-and- $q$, and $p$-and-not- $q$. The latter two conjunctive statements were accompanied by the clarification "both events must happen for you to win the money placed on this bet" (p. 204). In this betting context, putting more money on $p$-and- $q$ and $p$-and-not- $q$, respectively, than on $p$ is tantamount to violating the conjunction rule. Bonini et al. observed such bets in two out of five tasks ( $p$-and- $q$ versus $p$ ), and in one out of five tasks ( $p$ and-not- $q$ versus $p$ ), respectively. Did the clarification reduce the ambiguity of the and connective? Because the experiment did not include a control condition omitting this clarification, its impact is difficult to determine. When measured in terms of the rate of conjunction violations, the clarification may indeed have fostered reasoning in accordance with the conjunction rule: in merely four out of ten cases, the sum of money wagered on $p$-and- $q$ and $p$-andnot- $q$, respectively, significantly exceeded that wagered on $p$ (see Table 2 in Bonini et al.). In terms of the authors' more radical conjunctive dominance principle, which deems putting any money on the conjunctive statements as irrational, the average rate of violations was nearly twice as high, $76 \%$ (see Table 3 in Bonini et al.). ${ }^{2}$ In light of these results, directly gauging people's understanding of the and connective seems pertinent.

\section{Experiment 1}

\subsection{Method}

One hundred and nineteen participants were presented with a frequency representation of the Linda task and asked to provide an estimate for a single target item. The majority of respondents were students of two universities in Berlin, and about a third of respondents were professionals from different fields (e.g., architecture, medical care). The study was conducted at the Max Planck Institute for Human Development, Berlin. Respondents were randomly assigned to one of three conditions, and were paid for their participation.

Across the three groups, people first read Linda's personality sketch and then judged one of three target events:

Linda is 31 years old, single, outspoken, and very bright. She majored in philosophy. As a student, she was deeply concerned with issues of discrimination and social justice, and also participated in anti-nuclear demonstrations. Suppose there are 100 women like Linda. How many of them are

Bank tellers (Group 1: $n=30$ ).

Bank tellers and active feminists (Group 2: $n=59$ ).

Feminist bank tellers (Group 3: $n=30$ ).

The compound events in Groups 2 and 3 were those also investigated by Mellers et al. (2001), with "feminist bank tellers" avoiding the connective and altogether. The target "bank tellers" represents the unlikely single event.

\footnotetext{
2 According to Bonini et al. (2004, p. 201), conjunctive dominance suggests: "It is irrational to wager any sum of money on the truth of $p$-and$q$ if the money can be wagered on the truth of $p$ instead [except in the degenerate case where $\operatorname{Prob}(p$-and-not- $q)=0]$."
} 
Subsequent to their estimates, people in all groups were asked to shade the area in a Venn diagram that corresponded to the quantity they had just estimated. The Venn diagram consisted of two partly overlapping circles (one of which was labeled "bank tellers." the other "active feminists"), showing that the two groups have elements in common, but that both groups also have objects not included in the other group.

\subsection{Results}

Table 1 shows the average and median frequency estimates for the three target events, with standard errors (95\% confidence interval) in parentheses. Of initial interest was whether the average estimate for each conjunction was significantly greater than the average frequency estimate for the unlikely target item. Tests were done with an alpha level of 5\%, and significant effects are shown in bold. As in Mellers et al.'s (2001) three studies, conjunction effects occurred with the phrase "bank teller and active feminist." In contrast to Mellers et al. (Experiment 1), the conjunction effect did not occur with "feminist bank teller." One candidate reason is that unlike their experiment the current study did not include a filler item (see the discussion in Mellers et al. for the impact of filler items).

How did people understand the conjunction that they had just judged, and did estimates differ as a function of their understandings? Table 1 also shows the separate estimates for different understandings of and, as inferred from respondents' shadings of the Venn diagram. First, of the 59 participants in Group 2 who responded to the "bank tellers and active feminists" conjunction 14 (24\%) shaded the union of sets and 45 (76\%) shaded the intersection of sets. The estimates of these two groups differed enormously. Those who reported a union reading estimated, on average, that 59 out of 100 people like Linda are bank tellers and active feminists. When respondents' reported reading assumed a set interpretation, the average estimate was about 10 out of 100 on the intersection reading. In fact, the latter estimate is no longer significantly different to the average estimate for the unlikely target event (Table 1$)^{3}$

Another observation was indicative of the ambiguity of the "bank tellers and active feminists" conjunction. About half of respondents in Group 2 (27 of 59) explicitly asked the experimenter - during the estimation task - how the "and" was meant to be understood. The experimenter instructed them to assume the interpretation that appears most appropriate to them. Ambiguity was not an issue in Group 3, whose members judged "feminist bank tellers. With no exception, all 30 respondents marked the intersection of sets (or parts of the intersection to indicate that they thought the actual intersection was smaller than that implied by the graph).

\footnotetext{
3 Respondents provided estimates for only one of three target events. Therefore, it is not likely that people in the Venn diagram responded in such a way as to legitimize their estimate, as one reviewer suggested. Not having estimated the single event and the conjunction, no mistake had occurred. Consequently, it is not possible that participants' shading of the Venn diagram was an attempt to rectify their earlier judgment.
}

Table 1

Average and median frequency estimates in Experiment 1

\begin{tabular}{lll}
\hline Items & \multicolumn{2}{c}{ Frequency estimate } \\
\cline { 2 - 3 } & Mean & Median \\
\hline Bank tellers $(n=30)$ & $7.1(3.3)$ & 5 \\
Feminist bank tellers $(n=30)$ & $8.4(4.6)$ & 4 \\
Bank tellers and active feminists $(n=59)$ & $\mathbf{2 1 . 8}(10.9)$ & 7 \\
$\begin{array}{lll}\text { Bank tellers and active feminists interpreted } \\
\quad \text { as an intersection }{ }^{\mathrm{a}}(n=45)\end{array}$ & $10.2(5.0)$ & 5 \\
$\begin{array}{c}\text { Bank tellers and active feminists interpreted } \\
\quad \text { as a union }{ }^{\mathrm{a}}(n=14)\end{array}$ & $\mathbf{5 9 . 1}(14.0)$ & 68 \\
\hline
\end{tabular}

Note. 95\% confidence intervals are in parentheses. Boldface indicates a significant difference, relative to "bank tellers" $(p<.05)$.

a Based on respondents' answers in the Venn diagram task.

To conclude, these results suggest that the conjunction "bank tellers and active feminists" struck about half of respondents in Group 2 as ambiguous; for the other half, it referred to an intersection of events. Among the first group, about half (14 of 27) understood the statement "bank tellers and active feminists" as referring to the union rather than the intersection of sets, as indicated by their responses to the Venn diagram. The estimates of these respondents were about six times greater than estimates made by those who read the compound statement as referring to an intersection, causing the appearance of a conjunction effect.

In the next experiment, we investigate whether the combination of an and-conjunction that avoids ambiguity and an estimation response mode that avoids ambiguities triggered by a ranking response mode yields frequency estimates that conform to the conjunction rule.

\section{Experiment 2}

It would be plainly wrong to claim that recent research on violations of the conjunction rule has paid no attention to the ambiguity of and. Bonini et al. (2004) and Tentori, Bonini, and Osherson (2004), for instance, discussed this issue extensively. Notwithstanding their prudent treatment of semantic and pragmatic ambiguities in general they found numerous judgments that violated the conjunction rule. In what follows, we consider Tentori et al.'s (2004) Experiment II in more detail.

One of the two problems that respondents saw in Tentori et al.'s (2004) Experiment II is the Scandinavia task. It read:

The Scandinavian peninsula is the European area with the greatest percentage of people with blond hair and blue eyes. This is the case even though (as in Italy) every possible combination of hair and eye color occurs. Suppose we choose at random 100 individuals from the Scandinavian population. Which group do you think is the most numerous? (Check your choice.)

Individuals who have blond hair.

Individuals who have blond hair and blue eyes.

Individuals who have blond hair and do not have blue eyes.

The Scandinavia task has several interesting properties: first, by inserting the statement "Individuals who have blond hair and do not have blue eyes, it discourages 
respondents to read "Individuals who have blond hair" $(\mathrm{X})$ as " $X$-and-not- $Y$ " a conversational implicature that was suggested by a number of researchers in, for instance, the Linda task (e.g., Dulany \& Hilton, 1991; Politzer \& Noveck, 1991). Second, the Scandinavia task avoids the ambiguity of "probability" by asking for a frequency judgment. Frequency judgments have been found to reduce violations of the conjunction rule. ${ }^{4}$ Third, by introducing what they referred to as "implication questions" Tentori et al. (2004) also made the case that a majority of their participants understood that $X$-and- $Y$ implies $X$ but nevertheless attached greater probability to the former compared with the latter.

In light of this carefully phrased problem, can the finding that $46 \%$ of respondents (Tentori et al., 2004, Table 1) selected "individuals who have blond hair and blue eyes" as the most numerous group be explained other than by Tentori et al.'s conclusion of the existence of "a genuine (and elementary) error in reasoning about chance" (p. 475)? One possible alternative is related to another key property of the Scandinavia task: unlike in many other investigations of frequency representations, respondents were not instructed to estimate the groups' frequencies but to select the most numerous one.

By instructing them to select the most numerous group, respondents were implicitly asked to rank the most numerous group highest, and all other groups lower. Hertwig and Chase (1998) observed across several studies that people are much more likely to adhere to the conjunction rule when asked for probability estimates rather than probability ranks. Does their observation also generalize to frequency judgments as elicited in the Scandinavia task? According to a result reported by Hertwig (1995), the answer is yes. He studied the following frequency representation of the Linda task:

In an opinion poll, the 200 women selected to participate have the following features in common: They are on average 30 years old, single, very bright. []... How many of the women in the opinion poll sample will be found in the following groups? Please rank order the groups according to their frequency.

Bank tellers.

Active feminists.

Bank tellers and active feminists.

In this representation, 21 of 25 (84\%) participants violated the conjunction rule, compared with only 3 out of 23 (13\%) participants in a representation that required respondents to estimate frequencies. Note that some of this 71 percentage point difference is likely to be due to Hertwig (1995) using an and-conjunction within a nounnoun combination, giving rise to a union interpretation. It was this very finding that led to Hertwig's (1997) investi-

\footnotetext{
${ }^{4}$ Different explanations for the frequency effect in the conjunction problems have been proposed: Fiedler (1988) and Hertwig and Gigerenzer have argued that the term "frequency" (or how many, numerosity, etc.) narrows down the spectrum of possible interpretations to meanings that follow mathematical probability. In contrast, Kahneman and Tversky (1996) and Sloman, Over, Slovak, and Stibel (2003) have argued that the frequency effect results from the impact of extensional cues that are more salient in frequency formats.
}

gation of the ambiguity of and. The current Experiment 1 and the observation that about a quarter of people arrived at a union reading (see the Venn diagram task) suggest that the large difference in violations of the conjunction rule between Hertwig's (1995) two frequency representations may partly be due to the ambiguity of the and connective and partly be owed to the difference in response mode (ranking versus estimation). In fact, Sloman et al. (2003) found - using a who connective - that $69.9 \%$ participants violated the conjunction rule in a frequency ranking, relative to $33.3 \%$ in a frequency estimation version of the Linda task (for similar findings see also Wedell \& Moro, 2008).

Why does response mode matter? Sloman et al. (2003) suggested that "ranking would lead to more conjunction errors, because it forces a choice between the critical statements" (p. 306). Therefore, some people may violate the conjunction rule when they are forced to choose between two alternatives that they consider equally probable (frequent). Hertwig and Chase (1998) offered a theoretical account for the role of response mode. Their account, however, is restricted to probability judgments and cannot be applied to frequency judgments. Finally, Hertwig (1995) suggested that ranks are almost always assigned to categories that are mutually exclusive. Think, for instance, of rankings such as the Forbes' list of the world's billionaires, or the Shanghai academic ranking of world universities, or medal ranks in sports events. Therefore, the instructions either to "Please rank order the groups according to their frequency" (Hertwig, 1995) or "Which group do you think is the most numerous?" (Tentori et al., 2004) may have triggered participants to assume that some of the groups are mutually exclusive. In fact, the " $X$-and-not- $Y$ " conversational implicature, suggested in Linda-like tasks (e.g. Politzer \& Noveck, 1991), could be seen as people interpreting the $X$ and $X$-and- $Y$ statements as exclusive by extending the $X$ statement to $X$-and-not- $Y$. As described above, however, Tentori et al. did discourage such readings.

Beyond these ambiguities, the request for rankings also raises issues as to whether people's responses are indeed fallacious. In his review of Kahneman Slovic, and Tversky's (1982) Judgments Under Uncertainty: Heuristics and Biases, the philosopher of probability theory Isaac Levi (1985; see also Levi, 2008, for his notion of evidential support), for instance, made exactly this case in the context of the Linda task that required a ranking of probabilities:

But when someone is asked to rank propositions relative to data, it is quite easy for that person to construe his or her task to be to determine the grades of acceptability of the propositions on the data - i.e. to determine maximising probability. It is well known that evidence often supports (in the maximising sense) stronger hypotheses better than weaker ones. [...] If experimental subjects are assessing maximising probability as they might naturally be expected to be in the Linda problem, they commit no conjunction fallacy (p. 338).

Regardless of what one thinks about this line of argument, one can forestall this objection and the ambiguities of the ranking response mode by asking respondents in the Scandinavia task to estimate the frequency of each 
group rather than to select the most numerous group. That is exactly what we did in Experiment 2. Moreover, we, like Tentori et al. (2004), used a conjunctive statement in which the and connective combines two properties ("blond hair and blue eyes"), which together characterize one group of people. In contrast to the conjunction "bank tellers and active feminists" (Experiment 1), this conjunction does not represent two sets of people; thus, it avoids the ambiguity of whether it denotes the union (addition) or the intersection of both sets.

In sum, we investigated whether the occurrence of violations of the conjunction rule in the Scandinavia task would be reduced if (a) the and connective is not (or is less) ambiguous (compared to Experiment 1), and (b) respondents are asked to estimate rather than rank frequencies.

\subsection{Method}

Seventy-six native German speakers, who fittingly attended a summer camp in Sweden, participated in Experiment 2. About a fourth of participants were students from such varied disciplines as computer science, physics, medicine, business administration, educational science, and history; two-thirds were professionals with an academic degree. Their average age was about 30 years (range 1950 years). The experimental sessions were run in small groups of 3-10 participants in a quiet room. Each participant read the following version of the Scandinavia task, and provided four frequency estimates:

The Scandinavian peninsula is the European area with the greatest percentage of people with blond hair and blue eyes. This is the case even though (as in Germany) every possible combination of hair and eye color occurs. Suppose we choose at random 100 individuals from the Scandinavian population. How many of them have:

Blond hair and blue eyes: individuals have blond

hair and blue eyes.

Blue eyes: individuals have blue eyes.

Blond hair: individuals have blond hair.

Blond hair and not blue eyes: individuals have

blond hair and not blue eyes.

The order of the four targets was varied randomly across respondents. Note we added another event, "blue eyes," providing us with the opportunity of studying the possible occurrence of two violations of the conjunction rule. Judging from previous results, estimating both single events, relative to just one, does not to appear to affect the prevalence of violations of the conjunction rule (see Tversky \& Kahneman, 1983; Hertwig \& Gigerenzer, 1999).

\subsection{Results}

Table 2 shows the average and median frequency estimates for the four events. Averaged across all participants, the average frequency estimate for the conjunction "blond hair and blue eyes" was significantly smaller than for the single events "blue eyes" and "blond hair," respectively. The conjunction "blond hair and not blue eyes" received
Table 2

Average and median frequency estimates in Experiment 2

\begin{tabular}{llll}
\hline Items & Type of Item & \multicolumn{2}{l}{ Frequency estimates } \\
\cline { 3 - 4 } & & Mean & Median \\
\hline Blue eyes & Single event & $\mathbf{4 7 . 8 ( 4 . 6 )}$ & 50 \\
$\begin{array}{l}\text { Blond hair } \\
\begin{array}{c}\text { Blond hair and } \\
\text { blue eyes }\end{array}\end{array}$ & $\begin{array}{l}\text { Single event } \\
\text { Conjunction of events }\end{array}$ & $\mathbf{5 5 . 6 ( 4 . 5 )}$ & 60 \\
$\begin{array}{c}\text { Blond hair and } \\
\text { not blue eyes }\end{array}$ & $\begin{array}{l}\text { Conjunction that } \\
\text { discourages the }\end{array}$ & $\mathbf{2 9 . 9}(\mathbf{4 . 7})$ & 25 \\
& $\begin{array}{l}\text { X-and-not- } Y \\
\text { implicature (see text) }\end{array}$ & & \\
\hline
\end{tabular}

Note. 95\% confidence intervals are in parentheses. Boldface indicates a significant difference, relative to the "blond hair and blue eyes" $(p<.01)$.

by far the lowest frequency estimates. In addition, 63 of $76(83 \%)$ respondents estimated each of the constituents to be more frequent than the "blond hair and blue eyes" conjunction. Among the remaining 13 (17\%) participants, 9 (12\%) estimated one of the constituents to be higher than this conjunction, and 4 (5\%) thought both constituents to be more frequent than the conjunction. Across all 152 frequency estimates for the two constituents (76 respondents times 2 estimates), 135 estimates (89\%) were consistent with the conjunction rule; merely $11 \%$ of those judgments violated the conjunction rule.

To conclude, our results are strikingly different from those observed by Tentori et al. (2004) who reported that $61 \%$ (versus $17 \%$ in our study) of their participants violated the conjunction rule in their frequency representation of the Scandinavia task (in their Table 1, 61\% represents those judgments in which $X$-and- $Y$, and $X$-and-not- $Y$ is estimated larger than $X$ ). We showed that the combination of a relatively unambiguous and-conjunction (combination of two properties that together describe one group of individuals rather than the combination of two sets of individuals) and a frequency estimation mode resulted in a majority of respondents providing judgments that conformed to the conjunction rule. In our view, these findings do not call into question the results involving frequency ranks found in the careful experiments by Tentori et al., who paid much attention to the existence of semantic ambiguities (by avoiding the word "probable") and pragmatic inferences (by including a $X$-and-not- $Y$ statement). The significance of the results in Experiment 2 lies in demonstrating anew the feebleness of violations of the conjunction rule that appear to be a function of myriad variables including response format (probability versus frequency), response mode (ranks versus estimates), and the presence or absence of filler items (Mellers et al., 2001), and of semantic and pragmatic ambiguities.

\section{The and in causal conjunctions}

As pointed out before, Tversky and Kahneman (1983) distinguished between two task structures, the $M \rightarrow A$ paradigm and the $A \rightarrow B$ paradigm. Originally, it was predominantly the first paradigm that attracted researchers' imagination, and the Linda task evolved as its archetypical representative. Recently, however, the $A \rightarrow B$ paradigm has 
also been investigated (e.g., Sides, Osherson, Bonini, \& Viale, 2002; Bonini et al., 2004). According to Tversky and Kahneman, violations of the conjunction rule in this paradigm occur because the "added event, A, provides a plausible cause or motive for the occurrence of B" (p. 305). For illustration, consider Tversky and Kahneman's study involving professional analysts. They read one of the following two statements, and estimated their probability:

A complete suspension of diplomatic relations between the USA and the Soviet Union, sometime in 1983.

A Russian invasion of Poland, and a complete suspension of diplomatic relations between the USA and the Soviet Union, sometime in 1983.

Respondents judged the probability of the conjunction significantly more probable than the single event, violating the conjunction rule. Sides et al. (2002) highlighted the importance of this paradigm by arguing that "ambiguity surrounding the word and seems not to arise when it is used to connect propositions rather than categories. Thus, the sentence 'IBM stock will rise tomorrow and Disney stock will fall tomorrow' seems immune to a disjunctive reading" (p. 193). Although we agree that no union interpretation is invited, we do not agree that and in causal conjunctions is necessarily read as a conjunction of events.

To appreciate this objection, let us consider (6) from Sweetser (1990, pp. 87, 88):

(6) Why don't you want me to take basketweaving again this quarter?

Answer: Well, Mary got an MA in basketweaving, and she joined a religious cult.

According to Sweetser, (6) seems to be saying "that one concludes the likelihood of cult-joining from the knowledge that a person has a basketweaving MA" (p. 88). That is, the "answer" invites the conclusion that getting an MA in basketweaving caused Mary to join a religious cult. Similarly, Levinson (1995) posited that conjunctions can be "presumptively enriched to suggest sequential occurrence of events and, further, intention and causality" (p. 101).

In other words, according to these linguists and-conjunctions can convey and imply a causal relationship between the states of affairs described by the conjuncts. For example, take utterances (7) and (8):

(7) Jackie announced her intention to marry Ari and the rumors started flying.

(8) The lights were off and I couldn't see.

Both sentences can be understood as conveying "extra" information beyond what is explicitly expressed in the two conjuncts. As Schmerling (1975) pointed out, (7) “implies that Jackie's announcement was the direct cause of rumors" (p. 226), and in (8) the state of the environment, no light, is the reason for the state expressed in the second conjunct.

The fact that the and-conjunction can convey a causal meaning matters for the conjectured reality of the conjunction fallacy. If people infer a causal meaning, and therefore, wittingly or unwittingly, judge a conditional probability - for instance, the probability of a complete suspension of diplomatic relations between the USA and the Soviet Union given a Russian invasion of Poland rather than a conjunctive probability, their estimates will no longer violate the conjunction rule. The conditional probability may very well exceed the judged probability of the effect-conjunct. This diagnosis, however, would only be appropriate if respondents did indeed read the conjunctive event as a cause-effect event.

Do respondents read and-conjunctions as a statement about a cause-event relationship? To address this question, let us turn to Sides et al.'s (2002) experimental stimuli. They presented participants with pairs of events, and asked them to mark either the one "most likely to occur" or the one they "would like to bet on." One representative pair of events included, for instance:

$[X]$ The percentage of adolescent smokers in Texas will decrease at least 15\% from current levels by September 1, 1999.

$[Y \wedge X]^{5}$ The cigarette tax in Texas will increase by $\$ 1.00$ per pack and the percentage of adolescent smokers in Texas will decrease at least 15\% from current levels by September 1, 1999.

Sides et al. (2002) observed a high rate of violations of the conjunction rule across four experiments - findings they interpreted as demonstrating the "reality of the conjunction fallacy." But is it necessarily a fallacy to select the conjunctive event as the one most likely to occur? In our view, the answer depends on how respondents understood the conjunction. Let us consider the conjunctive statement in more detail.

The statement involves a supposition of real-world causation. In fact, Sides et al.'s (2002) explicit intention was to construct items such that one conjunct represents a plausible cause for the other conjunct. In their words, "In the fallacy items, the conjunct $Y$ was designed to enhance the plausibility of $X$ (i.e., $\operatorname{Pr}(X \mid Y)>\operatorname{Pr}(X)$, in the authors' opinion)" (p. 193). If participants inferred that the conjunctive statement invites the reasonable conclusion that the decrease in the percentage among adolescent smokers is caused by the increase in cigarette tax, it would not be implausible to suspect that they judged the conditional probability $p$ (effect|cause), rather than the conjoint probability $p$ (cause and effect). Because $p$ (effect|cause) can exceed $p$ (effect), participants do not commit a fallacy when they bet on the event "effect given a cause" rather than on the event "effect." Note that a similar argument would apply if people had inferred a temporal order between conjuncts. A temporal relationship between two events is typically not reversible and suggests that the relationship between the conjuncts will not be interpreted in terms of a set inclusion.

\footnotetext{
${ }^{5}$ In Table 1 of Sides et al. (2002), the authors used the notation $[Y \wedge X]$, suggesting that they assume the natural language and to be read as a logical operator.
} 


\section{Experiment 3}

Experiment 3 is designed to investigate how people read the cigarette-tax task constructed by Sides et al.'s (2002). To do this we make use of Levinson's (1983) analysis of and-conjunctions. He predicted that people read (9):

(9) He turned on the switch and the motor started

in a way that "is as 'strong' (informationally rich) as the world allows - and read in the following relations between two conjoined clauses wherever possible:

Given $p$ and $q$, try interpreting it as:

(i) ' $p$ and then $q$ '; if successful try:

(ii) ' $p$ and therefore $q$ '; if successful try also:

(iii) ' $p$, and $p$ is the cause of $q$ '." (p. 146)

If people do indeed informationally enrich clauses conjoined with and in the ways Levinson (1983) suggested which is not unlikely, given that the experimental statements in Sides et al. (2002) were explicitly constructed to convey cause-effect relationships - then people may reasonably judge the cause-effect relationship to be more probable than the effect per se. In contrast, those people who report having read the conjunctive event in a noncausal way should, unless their reasoning conflicts with the conjunction rule, judge it to be less probable than the single event. Experiment 3 investigates the link between people's reading of the conjunctive event and their probability judgments.

\subsection{Method}

One hundred and thirty students from the Free University, Berlin, participated in the experiment. It was conducted at the Max Planck Institute for Human Development, Berlin. Participants received a flat fee for their participation. Students were presented with a German adaptation of the cigarette tax items investigated by Sides et al. (2002). As in that study, 80 of the 130 students were asked to mark the more probable of the following two statements; their order was randomized:

$[X]$ The percentage of adolescent smokers in Germany decreases at least $15 \%$ from current levels by September 1, 2003.

[X-and- $Y]$ The tobacco tax in Germany is increased by 5 cents per cigarette and the percentage of adolescent smokers in Germany decreases at least 15\% from current levels by September 1, 2003.

After participants provided their probability judgment, they were asked to imagine four readers of the local newspaper who encountered the $X$-and- $Y$ statement in an article. They also learned that the four readers reproduced this statement in a later conversation with their colleagues at work. Participants were then asked to imagine themselves in the role of a reader who relates the newspaper statement to colleagues. Assuming this role, they were asked how they would have reproduced the original statement. Specifically, they were required to rank order the reproductions of the four readers according to how well they described their understanding of the $X$-and- $Y$ statement. Table 3 lists the readers' reproductions, of which three are designed according to Levinson's (1983) informational enrichments of and-conjunctions. Note that we also provided the original statement in the choice set of interpretations to give respondents the chance of selecting an understanding that is exactly identical to the original statement. If we nevertheless found that (some) people selected other readings as closer to their understanding of the original statement, this would provide strong evidence that the original statement invited causal or temporal readings. The order of the four reproductions was randomly mixed to avoid order effects. Out of 130 students 50 were asked only to imagine the four readers of the local newspaper, without providing a probability judgment. In this way, we could control whether people's reports of how they comprehended the conjoined clauses were in any way affected by the preceding probability judgment.

\subsection{Results}

Consistent with the results of Sides et al. (2002), most participants, 64 of 80 (80\%), selected the $X$-and- $Y$ statement as the more probable, violating the conjunction rule. How did they report their understanding of the $X$-and- $Y$ statement? More than half, 35 out of 64 (55\%), selected conjoined clauses involving and then, and therefore, and and $p$ is the cause for as those that described their understanding best. About a third, 21 out of those 64 participants who violated the conjunction rule, rated the conjoined clause with and as the one that represented their understanding least. Of the 80 respondents, 16 (20\%) selected the $X$-statement as the more probable and thus did not violate the conjunction rule. How did they report having understood the $X$-and- $Y$ statement? All but two participants (14 of $16,88 \%$ ) indicated that their understanding was best characterized by the conjoined clause which used and. That is, people whose judgment was consistent with the conjunction rule were much more likely to choose the conjoined clause using and as the statement that best characterized their understanding than were people who

Table 3

The four readings investigated in Experiment 3

\begin{tabular}{ll}
\hline and & The tobacco tax in Germany is increased by 5 cents per \\
cigarette and the percentage of adolescent smokers in \\
Germany decreases at least $15 \%$ from current levels by \\
September 1,2003 . \\
The tobacco tax in Germany is increased by 5 cents per \\
cigarette and then the percentage of adolescent smokers \\
in Germany decreases at least 15\% from current levels by \\
and then \\
September 1,2003 . \\
The tobacco tax in Germany is increased by 5 cents per \\
cigarette and therefore the percentage of adolescent \\
smokers in Germany decreases at least $15 \%$ from current \\
levels by September $1,2003$. \\
The tobacco tax in Germany is increased by 5 cents per \\
cigarette and this increase is the cause for the \\
percentage of adolescent smokers in Germany \\
decreasing at least $15 \%$ from current levels by September \\
1, 2003.
\end{tabular}




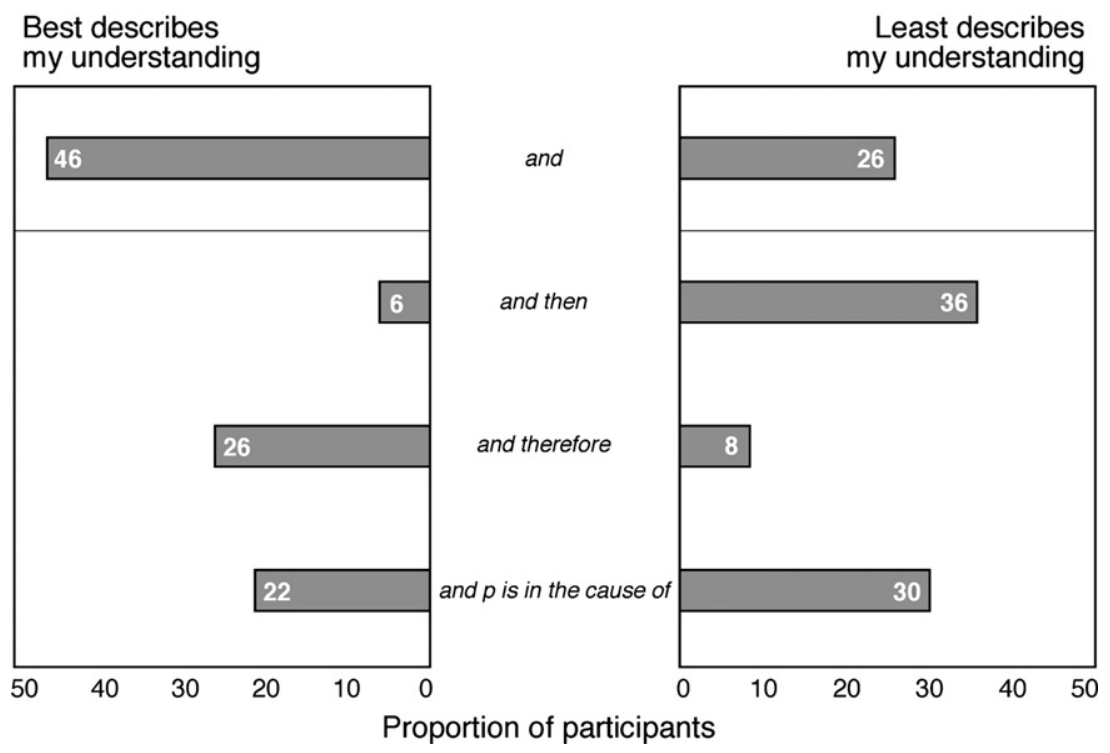

Fig. 1. Relative frequency with which participants $(n=50)$ ranked the conjoined clauses of as "best describes" (ranked highest) versus "least describes" (ranked fourth) their understanding of the target statement.

violated the conjunction rule (Fisher's Exact Test $p=.004$; one-tailed). Similarly, people who conformed to the conjunction rule were much more likely to choose the conjoined clauses and then, and therefore, and and $p$ is the cause for as statements that least characterized their understanding than were people who violated the conjunction rule (Fisher's Exact Test, $p=.03$; one-tailed).

Fig. 1 shows how often each of the four options was selected as the one that best and least described people's understanding by those 50 respondents who did not first provide probability judgments. More than half of the participants (54\%) indicated that their understanding was best described by causal and temporal relationships; $48 \%$ of participants ranked the two conjoined clauses that suggest a causal relationship, namely, and therefore, and $p$ is the cause for highest. Less than half (46\%) selected the and-conjunction as the one that best described their reading. In other words, the preceding probability judgment did not trigger the selection of causal and temporal readings. These readings also occurred among slightly more than half of the respondents who were just asked to consider how they would have reproduced the statement.

To conclude, as in Experiment 1, we found that people who do or do not violate the conjunction rule appear to operate on different understandings of the relationships conveyed by the and-conjunction. Experiment 3 indicates that in causal conjunctions people may infer causal and temporal relationships and therefore, we speculate, judge a conditional rather than a conjunctive probability. The final experiment tests this hypothesis directly.

\section{Experiment 4}

In Experiment 4, we investigate estimates of both conditional and conjunctive probability, elicited separately in two groups of respondents. If indeed, as Experiment 3 suggests, (some) people understand the tobacco-tax task as a cause-effect statement, and consequently, estimate the conditional probability, participants in the conditional probability group and the conjunctive-probability group will arrive at more or less identical estimates.

\subsection{Method}

Ninety-eight students participated in the study, which was conducted at the Max Planck Institute for Human Development, Berlin. Two groups of participants (each $n=49$ ) were presented with either the conjunctive or conditional version of the tobacco-tax task and a second one, the dental treatment task (see Table 4). They were asked to estimate the respective probability per task (on a percentage scale). The order of tasks was randomized. All students were paid a flat fee for participating. Up to five people took part in each session.

\subsection{Results}

Table 5 shows the mean estimates for the conjunctive and conditional versions of the tobacco-tax and dental-treatment tasks, respectively. Between tasks the probability estimates differed widely. A 5-cent increase in the tobacco tax was judged unlikely to result in a $15 \%$ decrease of adolescent smokers (conditional probability $=27 \%$ ). In contrast, people deemed it much more likely that the elimination of dental treatment from the public health insurance coverage would be tantamount to a person's teeth becoming a manifestation of his or her socio-economic status (conditional probability $=59 \%$ ) $-\mathrm{a}$ prominent concern in the public discourse about the German state-run health insurance system. Despite these differences, the average probability estimates per task were nearly identical, independent of whether respondents were asked to estimate a conjunctive or a conditional probability (Table 5 ). 
Table 4

The conjunctive and conditional representation of the tobacco-tax and dental-treatment tasks, respectively, in Experiment 4

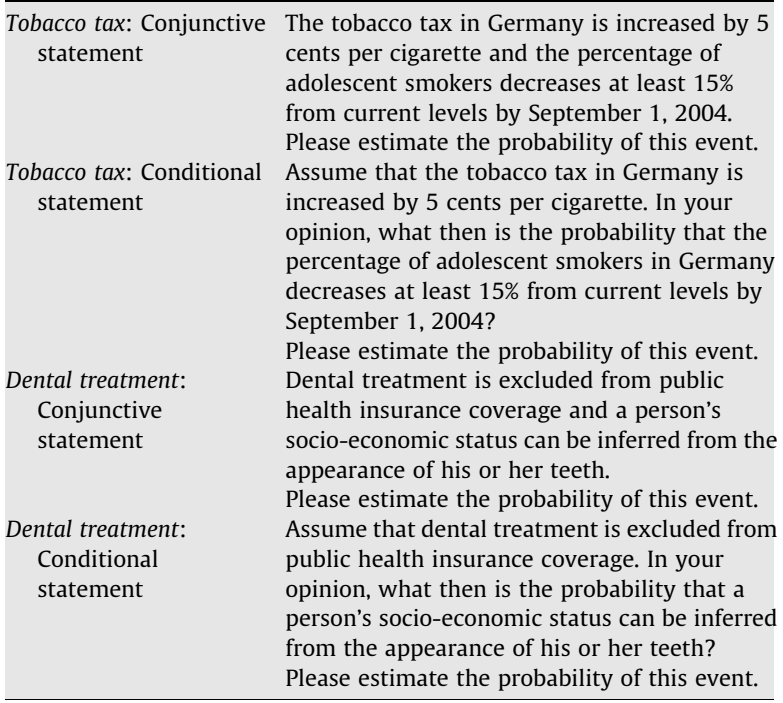

Table 5

Average and median probability estimates (in percentages) in Experiment 4

\begin{tabular}{llllll}
\hline \multirow{2}{*}{ Item } & \multicolumn{2}{l}{ Tobacco tax } & & \multicolumn{2}{l}{ Dental treatment } \\
\cline { 2 - 3 } & Mean & Median & & Mean & Median \\
\hline Conjunctive probability & $26.1(24.5)$ & 20 & & $59.6(28.3)$ & 60 \\
Conditional probability & $27(26.7)$ & 20 & & $59.1(27.9)$ & 70 \\
\hline
\end{tabular}

Note. Standard deviations of the mean are in parentheses. The mean estimates within each task are not significantly different.

Similar average estimates, however, can "hide" very dissimilar distributions of individual estimates (e.g., in terms of their variability). The same average value could represent the combination of extremely low and high estimates or the combination of very similar estimates. If the distribution of conjunctive and conditional probability estimates were quite different, people who responded to the conjunctive version would not be likely to have inferred a conditional interpretation. Fig. 2 plots the individual probability estimates for each of the 49 participants in the conjunctive and conditional probability groups, respectively. Like the means, the distributions look strikingly similar.

To conclude, independent of whether people responded to a conjunctive or conditional version of the tasks, people's average probability estimates and the distributions of individual estimates map onto each other. This is consistent with the hypothesis that (some) respondents in Experiments 3 and 4 and in Sides et al.'s (2002) experiments have interpreted the and-conjunctions in terms of causeeffect statements, and as a consequence judged conditional rather than conjoint probabilities.

\section{General discussion}

We have used three different methods to probe people's understanding of the and connective. In Experiment 1, we asked participants to shade the area in a Venn diagram that corresponded to the quantity that they had just estimated. In Experiment 3, we asked participants to imagine themselves in the role of a reader who relates a newspaper statement to colleagues, and to report how they would have reproduced the original statement. In Experiment 4, we asked participants in one group to estimate conjunctive probabilities and participants in another group to estimate conditional probabilities, and then compared both kinds of probability judgments.

Using these methods, we found first that some participants understood a plural noun-plural noun combination ("bank tellers and active feminists") as referring to a union rather than an intersection of events. Those people's frequency estimates were substantially higher than those of people who reported to have judged an intersection of events (Table 1). Second, we found that among those participants who violated the conjunction rule in their probability judgments more than half reported a temporal or causal reading of a cause-and-effect statement to reflect best how they understood the statement (Experiment 3); participants whose probability judgments were consistent with the conjunction rule nearly all reported not having read the statement in a temporal or causal sense. Third, we found that participants who were asked to judge the conjunctive probability, in response to a cause-and-effect statement, produced judgments that were interchangeable with those produced by participants who estimated a conditional probability, suggesting that people's conjunctive probabilities were conditional probability estimates (Table 5 and Fig. 2). Finally, in Experiment 2, we demonstrated that the use of an and-conjunction that avoids ambiguity in combination with an estimation response mode yielded a majority of frequency estimates that conformed to the conjunction rule.

These findings suggest that one can reliably measure people's understanding of the and-connective and the relationship conveyed between the conjuncts. Moreover, participants' reports of their understanding and their probability and frequency judgments were systematically linked. Our findings also suggest that participants' understandings of and can - to some extent - predict whether their estimates will violate the conjunction rule (in within-subjects designs) or yield conjunction effects (in between-subjects designs). In our view, these observations have implications for how these violations of the conjunction rule and conjunction effects should be evaluated. In what follows, we discuss these implications. We begin by turning again to how linguists think about and's ambiguity. Then, we turn to the relevance of our findings and linguists' insights to the ongoing debate on the conjunction fallacy, and their implications for human rationality.

\section{The ambiguity of and and semantic or pragmatic inferences}

Numerous linguists have emphasized that and-conjunctions can communicate a wide range of relationships between the states of affairs described by their conjuncts. Some linguists have taken the position that the natural 

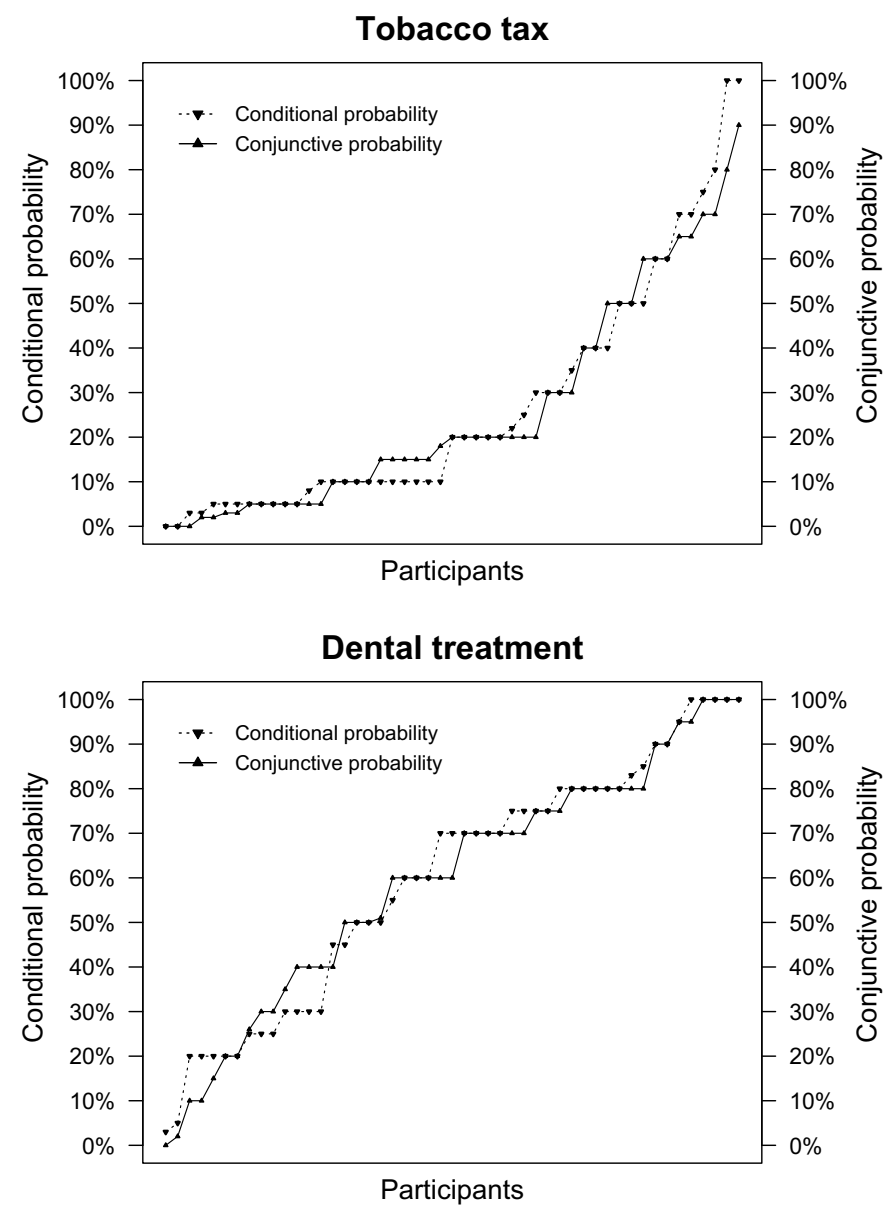

Fig. 2. Distribution of the individual's conjunctive and conditional probability estimates in the tobacco-tax task (top) and dental-treatment task (bottom), respectively. The estimates are rank-ordered according to their size, and the $x$-axis represents the 49 participants in the conjoint-probability group and the 49 different participants in the conditional-probability group, respectively.

language and should be analyzed independently of the logical operator $\wedge$ (e.g., Sweetser, 1990); other linguists have proposed that and has a minimal truth-functional semantics (e.g., Blakemore \& Carston, 2005). The key issue is on which levels do connotations arise that are not equivalent to the logical operator $\wedge$ ? To appreciate linguists' responses to this question, it helps to review a crucial distinction that linguists make: the distinction between two sources of utterance meanings, the semantic and the pragmatic (e.g., Carston, 1994). If a term has multiple, related meanings, and if those meanings are independent of particular contexts of use, linguists tend to diagnose semantic ambiguity. Take the word mouth as an example: it can refer to a facial aperture, the entrance to a cave, or the point where a river meets an ocean. Semantic ambiguity is ubiquitous. For instance, 85 of the 100 most frequent words in English have more than one meaning (Britton, 1978). Pragmatics, in contrast, takes as its domain all those aspects of utterance meaning that depend on an interaction of linguistic meaning (semantics) and contextual information: e.g., the question "Do you have coffee to go?" posed by a customer in a coffee shop carries the implied meaning "Sell me coffee to go if you can" (Levinson,
1983). Such implied meanings cannot be looked up in a dictionary.

Attempts to explain the different multiple meanings of and have gone both ways. Some have argued that its ambiguity is entirely a matter of semantics, and postulated that there are multiple ands in the language. Others have raised various objections to this lexical-polysemous analysis (see Carston, 1994, pp. 692-693). Rather than positing various discrete meanings, they have suggested that and represents "a unified semantic entity (with a range of systematically related uses)" (Sweetser, 1990, p. 91). Its ambiguity is thus not of semantic but of pragmatic nature; that is, "its single abstract sense will apply in different ways to the interpretation of the conjuncts, depending on the context" (Sweetser, 1990, p. 91).

Grice $(1975,1989)$ is the foremost proponent of the pragmatic approach. He proposed that the semantics of and are identical to the simple truth-functional semantics of the logical $\wedge$. Therefore, different meanings, rather than being features of the code itself, arise through interpretative processes that are guided and constrained by principles of reasonable communicative behavior. Take, for example, the conjoined utterances (10) and (11): 
(10) They got married and she got pregnant.

(11) She got pregnant and they got married.

In Grice's view, the differences in the interpretations of the two utterances do not arise from an extra, temporal sense of and, but from an interaction between its core meaning and a pragmatic maxim, here the maxim of orderliness, which instructs speakers to recount events in the order in which they occurred. Consequently, the nonequivalent temporal connotations in (10) and (11) are "linguistically communicated without being linguistically encoded" (Wilson \& Sperber, 1993, p. 6).

Numerous post-Gricean analyses of and have objected to various assumptions within Grice's account (e.g., Cohen, 1971). Endorsing a pragmatic approach, Sweetser (1990), for instance, doubted that the unified semantic core of and is equivalent to the logical $\wedge$, and Wilson and Sperber (1993) emphasized the role of the maxim of relevance and questioned the utility of maxims such as orderliness in explaining, for instance, causal connotations as in "Smile and the world smiles with you." Carston (1988) has argued that temporal and causal connotations of utterances should not be treated as implicatures but as pragmatically determined aspects of the proposition expressed. Kitis (2000), in contrast, proposed explaining coordinate conjunctions in terms of Minsky's frame theory, and suggested that connectives function as hypo-textual signals that activate stereotypical knowledge (frames or schemas).

Regardless of these different views, among linguists "it is well known that utterances of certain and-conjunctions communicate a range of temporal and consequence relations between the states of affairs described by their conjuncts" (Blakemore \& Carston, 2005, p. 569). These "relations" in turn, may either be part of the proposition expressed or arise only at the level of what was implicated. Relatedly, and can indicate the logical conjunction of propositions and functions as an indicator of addition (Hurford, 1987) - a fact that caused some linguists to propose the existence of two ands, one referring to intersection and one referring to union of sets. ${ }^{6}$ To conclude, the and connective can communicate a linguistically wide range of relationships between conjuncts. As a consequence, the speaker's use of and can require the recipient's interpretative effort, and interpretations other than the logical $\wedge$ are reasonable, and probably the rule rather than the exception.

\section{The conjunction fallacy: A misunderstanding about conjunction?}

In a series of studies, Noveck and Chevaux (2002) demonstrated that children appear less likely than adults to draw implicit meanings from the conjunction in statements that invert a series of events such as "Laurent went to the hospital and broke his ankle." In their view, this and related findings (e.g., Noveck, 2001) show that "a child's initial treatment of utterances ... is consistent with logical

\footnotetext{
6 Interestingly, if mathematicians were asked for a definition of and, they would probably also point to these two opposite meanings. Specifically, there are at least two mathematical symbols - namely " $\wedge$ " and "+" - which in mathematical colloquial language are referred to as and.
}

interpretations and that linguistic-pragmatic interpretations evolve with age" (p. 6). Here is the irony: children are not the superior logicians. They simply have not yet (or not to the same extent as adults) developed the ability to go beyond the explicitly stated. Only after children have successfully proceeded through their cognitive development will they be able to attend to and benefit from the semantic and pragmatic subtleties of natural language and social communication (see also Braine \& Rumain, 1981; Paris, 1973; Politzer, 1986; Sternberg, 1979). This very ability, however, can result in judgments that violate rules of probability theory or logic - rules that interpret connectives such as and or or in a purely logical sense.

Are such judgments that violate the conjunction rule in the kind of problems, as we studied them in Experiments 1, 3 and 4, instances of the conjunction fallacy? Some authors would emphatically argue yes. Tentori et al. (2004; see also Bonini et al., 2004), for instance, made this case in their article "The conjunction fallacy: A misunderstanding about conjunction":

Nor does it disarm the fallacy to claim that many people evaluate $\operatorname{Prob}(X$-and- $Y)$ via the conditional probability of $Y$ given $X \ldots$ Even if this claim is true, it merely indicates the reasoner's path to fallacy. Such use of conditional probability is compatible with assigning $X$-and- $Y$ a higher probability than the one assigned to $Y$, so conditional probability should not be used in this way by anyone wishing to conform to the probability calculus. Perhaps it will therefore be agreed that these data point to a genuine (and elementary) error in reasoning about chance (p. 475).

We disagree. To appreciate our view, consider again the Gricean framework that emphasizes that effective communication is built upon the mutual cooperation of sender and receiver. Lee (2006; see also Clark \& Schober, 1992) went even further and argued that in the experimental context

Experimenters shoulder a greater responsibility for clear communication because of the asymmetrical nature of experimenter-subject communication. Unlike ordinary conversation, the course and content of communication in experimental conditions are predetermined by only one conversant, namely, the experimenter. [...] experimenters are especially responsible for making "correct assumptions about the codes and contextual information that the audience will have accessible and be likely to use in the comprehension process" (p. 202).

Experiments 3 and 4 provide evidence that suggests that at least some people in Tentori et al.'s (2004) tobacco-tax task have inferred - in the process of comprehending the sentence - a causal meaning, and, therefore, appear to have estimated the conditional rather than the conjunctive probability. Is inferring a causal connotation in itself fallacious? In light of how and is used in natural language and the kind of connotations it can convey, a causal reading is by no means unreasonable.

We do not intend to claim that any violation of the conjunction rule can be explained in terms of semantic and pragmatic inferences regarding the and connective. In Study 3, we found that 29 of the 64 (45\%) respondents 
who violated the conjunction rule selected the conjoined clause involving and as the one that described their understanding best. Not knowing their reasons, this observation shows that there may be people who commit a genuine conjunction error. Yet, the complementary observation that more than half, 35 out of 64 (55\%), selected conjoined clauses involving and then, and therefore, and and $p$ is the cause for as those that described their understanding best suggests that, at the very least, estimates of the prevalence of genuine conjunction errors in previous studies are quite inflated.

\section{Conclusion}

The ability to make reasonable pragmatic inferences is a sine qua non of human intelligence, and people bring this intelligence into the laboratory. Any computer can mechanically apply the conjunction rule. In contrast, to winnow out the inappropriate readings of ambiguous words and statements, to infer the referents of pronouns and the meanings of polysemous words, and to fill in intended interpretations of what has been said is evidence of the remarkable linguistic abilities of humans. The juxtaposition is ironic: Whereas many judgment and decision-making researchers continue to interpret the outcome of semantic and pragmatic inferences as evidence of human irrationality (but for a very different view see McKenzie, 2004; Sher \& McKenzie, 2006), legions of computer scientists, linguists, mathematicians, engineers, and psychologists struggle to design artificial agents capable of making exactly those inferences. To many of those scientists, designing systems that can "process language as skillfully as we do will signal the arrival of truly intelligent machines" (Jurafsky \& Martin, 2000, p. 6).

\section{Acknowledgements}

Ralph Hertwig was supported by Swiss National Science Foundation Grant 100014-118283/1. We thank Gregor Caregnato for help in data collection and Laura Wiles for editing the manuscript.

\section{References}

Adler, J. E. (1984). Abstraction is uncooperative. Journal for the Theory of Social Behaviour, 14, 165-181.

Adler, J. E. (1991). An optimist's pessimism: Conversation and conjunction. Posnan Studies in the Philosophy of the Sciences and Humanities, 21, 251-282.

Blakemore, D., \& Carston, R. (2005). The pragmatics of sentential coordination with "and". Lingua, 115, 569-589.

Bonini, N., Tentori, K., \& Osherson, D. (2004). A different conjunction fallacy. Mind \& Language, 19, 199-210.

Braine, M. D. S., \& Rumain, B. (1981). Development of comprehension of "or": Evidence for a sequence of competencies. Journal of Experimental Child Psychology, 31, 46-70.

Britton, B. K. (1978). Lexical ambiguity of words used in English text Behaviour Research Methods and Instrumentation, 10, 1-7.

Carston, R. (1988). Implicature, explicature and truth-theoretic semantics. In R. Kempson (Ed.), Mental representation: The interface between language and reality (pp. 155-181). Cambridge, England: Cambridge University Press.

Carston, R. (1994). Conjunction and pragmatic effects. Encyclopaedia of language and linguistics. Oxford, England: Pergamon Press.
Clark, H. H., \& Schober, M. F. (1992). Asking questions and influencing answers. In Judith M. Tanur (Ed.), Questions about questions: Inquiries into the cognitive bases of surveys (pp. 15-48). New York: Russell Sage Foundation.

Dulany, D. E., \& Hilton, D. J. (1991). Conversational implicature, conscious representation, and the conjunction fallacy. Social Cognition, 9, 85-110.

Fiedler, K. (1988). The dependence of the conjunction fallacy on subtle linguistic factors. Psychological Research, 50, 123-129.

Gigerenzer, G. (1991). How to make cognitive illusions disappear: Beyond heuristics and biases. European Review of Social Psychology, 2, 83-115.

Gigerenzer, G. (1996). On narrow norms and vague heuristics: A reply to Kahneman and Tversky (1996). Psychological Review, 103, 592-596.

Grice, H. P. (1975). Logic and conversation. In P. Cole \& J. L. Morgan (Eds.), Syntax and semantics 3: Speech acts (pp. 41-58). New York: Academic Press.

Grice, H. P. (1989). Studies in the way of words. Cambridge, MA: Harvard University Press.

Hertwig, R. (1995). Why Dr. Gould's homunculus doesn't think like Dr. Gould: The "conjunction fallacy" reconsidered. Konstanz: Hartung-Gorre.

Hertwig, R. (1997). Judgment under uncertainty: Beyond probabilities. Berlin, Germany: Max Planck Institute for Human Development. Unpublished manuscript.

Hertwig, R., \& Chase, V. M. (1998). Many reasons or just one: How response mode affects reasoning in the conjunction problem. Thinking E Reasoning, 4, 319-352.

Hertwig, R., \& Gigerenzer, G. (1999). The conjunction fallacy revisited: How intelligent inferences look like reasoning errors. Journal of Behavioral Decision Making, 12, 275-305.

Hintikka, J. (2004). A fallacious fallacy? Synthese, 140, 25-35.

Hurford, J. R. (1987). Language and number: The emergence of a cognitive system. Oxford, England: Basil Blackwell.

Jurafsky, D., \& Martin, J. H. (2000). Speech and language processing. Upper Saddle Rivers, NJ: Prentice Hall.

Kahneman, D., \& Tversky, A. (1996). On the reality of cognitive illusions. Psychological Review, 103, 582-591.

Keenan, E. L., \& Faltz, L. M. (1985). Boolean semantics for natural language. Dordrecht: Reidel.

Kitis, E. (2000). Connectives and frame theory. The case of antinomial hypotextual 'and'. Pragmatics \&' Cognition, 8, 357-409.

Lang, E. (1991). Koordinierende Konjunktionen. In A. von Stechow \& D. Wunderlich (Eds.), Semantik. Semantics. Ein internationales Handbuch der zeitgenössischen Forschung (pp. 597-623). Berlin/New York: De Gruyter.

Lee, C. J. (2006). Gricean charity: The Gricean turn in psychology. Philosophy of the Social Sciences, 36, 193-218.

Leech, G., Rayson, P., \& Wilson, A. (2001). Word frequencies in written and spoken English: Based on the British National Corpus. London: Longman.

Levinson, S. (1983). Pragmatics. Cambridge: Cambridge University Press.

Levi, I. (1985). Illusions about uncertainty. British Journal for the Philosophy of Science, 36, 331-340.

Levi, I. (2004). Jaakko Hintikka. Synthese, 140, 37-41.

Levi, I. (2008). Degrees of belief. Journal of Logic and Computation. doi:10.1093/logcom/exmo96. Available online 4 March, 2008.

McKenzie, C. R. M. (2004). Framing effects in inference tasks - And why they are normatively defensible. Memory and Cognition, 32, 874-885.

Mellers, B., Hertwig, R., \& Kahneman, D. (2001). Do frequency representations eliminate conjunction effects? An exercise in adversarial; collaboration. Psychological Science, 12, 269-275.

Moldoveanu, M., \& Langer, E. (2002). False memories of the future: A critique of the applications of probabilistic reasoning to the study of cognitive processes. Psychological Review, 109, 358-375.

Noveck, I. A. (2001). When children are more logical than adults: Experimental investigations of scalar implicature. Cognition, 78 , $165-188$

Noveck, I. A., Chevaux, F. (2002). The pragmatic development of and, twenty-sixth annual Boston University conference on language development. Sommerville, MA: Cascadilla Press.

Paris, S. G. (1973). Comprehension of language connectives and prepositional logical relationships. Journal of Experimental Child Psychology, 16, 278-291.

Partee, B., \& Rooth, M. (1983). Generalized conjunction and type ambiguity. In R. Bäuerle, C. Schwarze, \& A. von Stechow (Eds.), Meaning, use and interpretation of language (pp. 361-383). Berlin: De Gruyter.

Politzer, G. (1986). Laws of language use and formal logic. Journal of Psycholinguistic Research, 15, 47-92.

Politzer, G., \& Noveck, I. A. (1991). Are conjunction rule violations the result of conversational rule violations? Journal of Psycholinguistic Research, 20, 83-103. 
Schmerling, S. (1975). Asymmetric conjunction and rules of conversation. In P. Cole \& J. Morgan (Eds.), Syntax and semantics. Speech acts (Vol. 3). New York: Academic Press.

Sher, S., \& McKenzie, C. R. M. (2006). Information leakage from logically equivalent frames. Cognition, 101, 467-494.

Sides, A., Osherson, D., Bonini, N., \& Viale, R. (2002). On the reality of the conjunction fallacy. Memory $\mathcal{E}$ Cognition, 30, 191-198.

Sloman, S. A., Over, D., Slovak, L., \& Stibel, J. M. (2003). Frequency illusions and other fallacies. Organizational Behavior and Human Decision Processes, 91, 296-309.

Sternberg, R. J. (1979). Developmental patterns in the encoding and combination of logical connectives. Journal of Experimental Child Psychology, 28, 469-498.
Sweetser, E. E. (1990). From etymology to pragmatics: Metaphorical and cultural aspects of semantic structure. Cambridge, MA: Cambridge University Press.

Tentori, K., Bonini, N., \& Osherson, D. (2004). The conjunction fallacy: A misunderstanding about conjunction? Cognitive Science, 28, 467-477.

Tversky, A., \& Kahneman, D. (1983). Extensional versus intuitive reasoning: The conjunction fallacy in probability judgment. Psychological Review, 90, 293-315.

Wedell, D. H., \& Moro, R. (2008). Testing boundary conditions for the conjunction fallacy: Effects of response mode, conceptual focus, and problem type. Cognition, 107, 105-136.

Wilson, D., \& Sperber, D. (1993). Linguistic form and relevance. Lingua, 90, $1-25$. 